\title{
The Transition of the Care Model
}

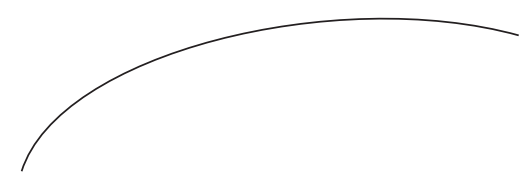

Over the last 50 years Brazil has experienced two processes of transition which have had a direct impact on the lives of its citizens: a demographic transition, with the elderly population growing at the expense of children and young people, and an epidemiological transition, with a reduced burden of infectious disease and an increase in chronic diseases, which are largely responsible for mortality and morbidity.

Life expectancy in the 1960s was 48, while the birth rate was four children per woman. Today, life expectancy is 78 years and the birth rate is 1.6 children. This has caused the number of elderly people to rise from $4.5 \%$ to $13 \%$ of the overall population over this period.

The result is that people are living longer, with a significant segment of the older population suffering from chronic diseases that require long-term care and which can lead to disability and functional limitation, making individuals completely dependent on the care of others.

Our health and social care model, however, remains the same as in the industrial era, structured in terms of scale and productivity but lacking personalization and individualization. The focus is on the disease, not the person.

The structure of the current health model is aimed at the treatment of disease based on diagnosis via laboratory and imaging tests, therapeutic procedures and drug therapy. There is no space for prevention and the system responds to health care needs with increasing numbers of consultations, doctors, specialists, emergency units, ambulance call-outs, hospitals and ICUs.

As we can already see, continuing in this way is unsustainable. By 2030, the elderly population will consume $46 \%$ of the hospitalization costs of the SUS, the Brazilian Public Health Service.

There is a disconnect between the objectives of health professionals and patients who want to live and preserve their functionality as much as possible despite their diseases and symptoms. This difference in priorities makes successful disease management impossible, further burdening the system.

Where do we go from here? Towards person-centered, individualized care, planned according to the living conditions of each individual and not just the disease they are suffering from. This is comprehensive health care. Care that is coordinated and integrated, irrespective of where it is provided. Care where the individual actively participates in decisions about how he or she should be treated, rather than acting simply as a patient who receives prescriptions and guidance on how to tackle diseases. 
People-centered care is responsive to the preferences, needs and values of the individual and ensures that these concepts guide all clinical decisions. It would represent a transition from a model where the health professional has the task of deciding the treatment of the patient, to a new model where he or she has the role of supporting the autonomy and individual choice of the person under his or her care.

We urgently need to begin the transition to a new model where listening prevails over the number of consultations and where the identification of health needs is as important as the diagnosis of diseases, providing clarity on the health situation of the individual rather than simply giving instructions. The focus of the health professional and the patient should not, at the end of the medical consultation, be simply the carrying out of exams and the prescription of medication, but the satisfaction and well-being of the person receiving care.

João Bastos Freire Neto President of the Brazilian Geriatrics and Gerontology Society 